\title{
ON THE CARDINALITY OF A TOPOLOGY
}

\author{
RUAN YONGBIN
}

(Communicated by Dennis Burke)

\begin{abstract}
Let $o(X)$ denote the cardinality of topology of a space $X$. I. Juhasz proves that $o(X)^{\omega}=o(X)$ for regular hereditarily paracompact spaces. We prove it for more general classes of spaces.
\end{abstract}

If $X$ is a topological space, let $o(X)$ denote the cardinality of its topology. A number of papers $[1,2,3]$ have investigated classes for which $o(X)=o(X)^{\omega}$. We generalize several of these results by proving

THEOREM 1. For every infinite hereditarily weakly $\theta$-refinable $T_{3}$ space $X, o(X)=$ $o(X)^{\omega}$.

A space $X$ is weakly $\theta$-refinable [4] if and only if each open cover $\mathscr{U}$ of $X$ has a refinement $\mathscr{V}=U_{n \in \omega} \mathscr{V}_{n}$ where each $\mathscr{V}_{n}$ is a discrete family of subsets of the space $\cup \mathscr{V}_{n}$.

If $\eta$ and $\mu$ are infinite cardinals and $\mu^{\prime}<\mu$ implies $\eta^{\mu^{\prime}}<\eta^{\mu}$ and $\eta^{\prime}<\eta$ implies $\eta^{\prime \mu}<\eta^{\mu}$, then $\eta^{\mu}$ is called a jump. By Theorem 0.2 of [2], if $\eta^{\mu}$ is a jump, $\mu$ is regular.

Since $X$ is $T_{3}$, by Theorem 4.3 of [2], if $\mu$ is an uncountable cardinal of cofinality $\omega$ and there is a discrete subset $S^{\prime}$ of $X$ with $\left|S^{\prime}\right|=\mu^{\prime}$ for all $\mu^{\prime}<\mu$, then there is a discrete subset $S$ of $X$ with $|S|=\mu$.

Before proving the theorem, we also need

LEMMA. If $\left\langle\kappa_{\alpha} \mid \alpha \in \gamma\right\rangle$ is a sequence of infinite cardinals, then there is an $S \subset \gamma$ with $\prod_{\alpha \in \gamma} \kappa_{\alpha}=\left(\sup _{\alpha \in S} \kappa_{\alpha}\right)|S|$.

Proof of Lemma. Enumerate the $\kappa_{\alpha}$ so that they form a nondecreasing sequence. Let $S \subset \gamma$ be of minimal order type so that $\prod_{\alpha \in \gamma} \kappa_{\alpha}=\Pi_{\alpha \in S} \kappa_{\alpha}$. Without loss of generality, suppose $S$ is infinite. Note that if $\beta<\sup S$, then $S-(\beta+1)$ has the order type of $S$, in particular $|S-(\beta+1)|=|S|$. So if $|S|=\lambda$ we have that $S=\lambda \circ \alpha$ for $\alpha=1$ or for some limit ordinal $\alpha<\lambda^{+}$. Split $S$ into $\lambda$ many cofinal disjoint sets $\left\{A_{\beta} \mid \beta \in \lambda\right\}$. Note that every $A_{\beta}$ is cofinal in $S$ and so

$$
\prod_{\delta \in A_{\beta}} \kappa_{\delta} \geqslant \sup _{\delta \in A_{\beta}} \kappa_{\delta}=\sup _{\alpha \in S} \kappa_{\alpha}=\kappa .
$$

Received by the editors January 6, 1986 and, in revised form, April 18, 1986 and July 28, 1986. 1980 Mathematics Subject Classification (1985 Revision). Primary 54A25, 04A30; Secondary 03 E05.

Key words and phrases. Hereditary paracompactness, weak $\theta$-refinability, weakly collectionwise normality. 
Hence

$$
\kappa^{\lambda} \leqslant \prod_{\beta \in \lambda} \prod_{\delta \in A_{\beta}} \kappa_{\delta}=\prod_{\alpha \in S} \kappa_{\alpha} ; \quad \text { so } \kappa^{\lambda}=\prod_{\alpha \in \gamma} \kappa_{\alpha}
$$

Proof of Theorem. Assume not and let $X$ be a counterexample; so $o(X)=\kappa<$ $\kappa^{\omega}$. Let $\lambda=\min \left\{\mu \mid \mu^{\omega}>\kappa\right\}$. Then $\operatorname{cf}(\lambda)=\omega$ and $\lambda \leqslant \kappa$.

For $x \in X$, let $\sigma(x, X)=\min \{o(U) \mid x \in U$ and $U$ is open $\}$ and $\sigma=\sigma(X)=$ $\sup \{\sigma(x, X) \mid x \in X\}$.

Note that there exists a finite subset $F \subset X$ such that $\sigma(X-F)<\lambda$. To see this assume the contrary and let $\left\langle\lambda_{n} \mid n \in \omega\right\rangle$ be a cofinal sequence in $\lambda$. At stage $n$ pick $x_{n} \in X-\left\{x_{k} \mid k \in n\right\}$ with $\sigma\left(x_{n}, X\right) \geqslant \lambda_{n}$. Since $X$ is regular there is a disjoint open family $\left\{U_{n} \mid n \in \omega\right\}$ with $x_{n} \in U_{n}$, hence $o\left(U_{n}\right) \geqslant \lambda_{n}$. So $o(X) \geqslant \Pi_{n \in \omega} o\left(U_{n}\right)$ $=\lambda^{\omega}>\kappa$, a contradiction.

Thus we can assume that $\sigma(X)<\lambda$ and that $o(X)=o(X-F)$ for all finite $F \subset X$ (recall that our original $X$ was hereditarily weakly $\theta$-refinable).

Let $\mathscr{U}$ be an open cover of $X$ such that each $U$ in $\mathscr{U}$ has $o(U) \leqslant \sigma$. Since $X$ is weakly $\theta$-refinable there is a refinement $\mathscr{V}=\bigcup_{n \in \omega} \mathscr{V}_{n}$ such that each $\mathscr{V}_{n}$ is a closed discrete family and $\bigcup \mathscr{V}_{n}=V_{n}$. Assume $\mathscr{V}_{n}=\left\{V_{n}^{\alpha} \mid \alpha \in \beta_{n}\right\}$ and $\beta_{n}$ is infinite for each $n \in \omega$. Then $\kappa=o(X) \leqslant \Pi_{n \in \omega} o\left(V_{n}\right)$. Now we derive a contradiction by showing that $\Pi_{n \in \omega} o\left(V_{n}\right)<\kappa$.

Fixing $n \in \omega$, we have $o\left(V_{n}\right)=\prod_{\alpha \in \beta_{n}} o\left(V_{n}^{\alpha}\right)=\sigma_{n}^{\mu_{n}}$ for $\sigma_{n}=\sup \left\{o\left(V_{n}^{\alpha}\right) \mid \alpha \in \beta_{n}\right\}$ and $\mu_{n}=\left|\beta_{n}\right|$ by the lemma. Since $o\left(V_{n}^{\alpha}\right) \leqslant \sigma$ for every $\alpha$, we have that $\sigma_{n} \leqslant \sigma$. Also $\sigma_{n}^{\mu_{n}}<\lambda$ since $o\left(V_{n}\right) \leqslant o(X)=\kappa$ and $\sigma_{n}^{\mu_{n} \cdot \omega}=\sigma_{n}^{\mu_{n}} \leqslant \kappa$ if $\mu_{n} \geqslant \omega$; otherwise $\sigma_{n}^{\mu_{n}} \leqslant \sigma$ $<\lambda$. Note that $\Pi\left\{\sigma_{n}^{\mu_{n}} \mid n \in \omega\right.$ and $\left.\sigma_{n}^{\mu_{n}}<\sigma\right\} \leqslant \sigma^{\omega}<\lambda$. So in order to show that $\prod_{n \in \omega} \sigma_{n}^{\mu_{n}}<\kappa$ we may assume that $\sigma \leqslant \sigma_{n}^{\mu_{n}}$ for all $n$. Then $\sigma_{n}^{\mu_{n}}=\sigma^{\mu_{n}}<\lambda$ (since $\left.\sigma_{n} \leqslant \sigma \leqslant \sigma_{n}^{\mu_{n}}<\lambda\right)$. Hence if $\mu=\sup _{n \in \omega} \mu_{n}, \Pi_{n \in \omega} \sigma_{n}^{\mu_{n}}=\sigma^{\mu}$. If $\sigma^{\mu}<\lambda$, then $\Pi_{n \in \omega} o\left(\mathscr{V}_{n}\right)^{\prime}<\kappa$ as desired.

So assume that $\sigma^{\mu} \geqslant \lambda$. Since $\sigma^{\omega}<\lambda, \mu$ is uncountable and, since $\sigma^{\mu_{n}}<\lambda$ for all $n, \operatorname{cf}(\mu)=\omega$. If $\eta=\min \left\{\alpha \mid \alpha^{\mu} \geqslant \lambda\right\}$, then $\eta^{\prime}<\eta$ implies $\eta^{\prime \mu}<\eta^{\mu}$ and $\mu^{\prime}<\mu$ implies $\eta^{\mu^{\prime}}<\eta^{\mu}$. So if $\eta$ is infinite, $\eta^{\mu}$ is a jump and $\mu$ is regular. This contradicts $\mu$ being uncountable and of cofinality $\omega$.

So it remains to show that $\eta$ is infinite if $\mu$ is. To show this, for each $n \in \omega$ and $\alpha \in \beta_{n}$ choose $x_{\alpha} \in V_{n}^{\alpha}$ and let $S_{n}=\left\{x_{\alpha} \mid \alpha \in \beta_{n}\right\}$. Since $S_{n}$ is discrete and $\left|S_{n}\right|=\mu_{n}$, there is a discrete $S \subset X$ of cardinality $\mu$. Thus $2^{\mu} \leqslant o(S) \leqslant o(X)=\kappa$. Since $\mu$ is infinite, $2^{\mu \cdot \omega}=2^{\mu} \leqslant \kappa$ while $\lambda^{\omega}>\kappa$, so $2^{\mu}<\lambda$. Since $2^{\mu}=\eta^{\mu}$ for finite $\eta$ and $\eta^{\mu} \geqslant \lambda$ by definition, $\eta$ is infinite.

Our proof gives the stronger

THEOREM 2. Let $X$ be regular space. If for any finite subset $F, X-F$ is weakly $\theta$-refinable, then $o(X)=o(X)^{\omega}$.

Since weak $\theta$-refinability is hereditary to $F_{\sigma}$-sets, $o(X)=o(X)^{\omega}$ also holds for 1st countable, $T_{3}$, weakly $\theta$-refinable spaces or 1 st countable, $T_{3}$ paracompact spaces.

A similar proof also yields a theorem, independently proved by I. Juhasz, that $o(X)=o(X)^{\omega}$ for all hereditarily weakly collectionwise Hausdorff spaces. 
In the end, I acknowledge my indebtedness to Zhou Hao-Xuan and Amer Beslagic for their help.

\section{REFERENCES}

1. I. Juhasz, Two set-theoretic problems in topology, Proc. 4th Prague Sympos. on General Topology (1976), Part A, Springer-Verlag, pp. 115-123.

2. Cardinal functions in topology-Ten years later, MCT 123, Mathematisch Centrum, Amsterdam, 1980.

3. E. K. Van Douwen and Zhou Hao-Xuan, The number of cozero-sets is an $\omega$-power, 1980.

4. H. R. Bennett and D. J. Lutzer, A note on weak $\theta$-refinability, General Topology Appl. 2 (1972), 49-54.

Department of Mathematics, University of Wisconsin, MADISON, Wisconsin 53706

Current address: Department of Mathematics, University of California-Berkeley, Berkeley, California 94720 